\title{
Das Interdisziplinäre Brustzentrum im HELIOS Klinikum Berlin-Buch
}

\author{
$\begin{array}{lll}\text { M. Untch } & \text { E. Keila } & \text { U. Kettritz } \\ & \text { b }\end{array}$ \\ T. Mairinger ${ }^{\mathrm{C}}$ \\ B. Fleige ${ }^{c}$ \\ S. Dresel ${ }^{\mathrm{d}}$ \\ R. Krempien ${ }^{\mathrm{e}}$ \\ A. Kretzschmar ${ }^{\text {h }}$ \\ P. Preussker \\ H. Lolla \\ H. Lüders ${ }^{g}$ \\ J. Wildberger ${ }^{b}$ \\ E. Schuleri \\ a Klinik für Gynäkologie und Geburtshilfe und Interdisziplinäres Brustzentrum, \\ b Institut für Röntgendiagnostik und Interdisziplinäres Brustzentrum, \\ ${ }^{c}$ Institut für Pathologie und Interdisziplinäres Brustzentrum, \\ ${ }^{d}$ Klinik für Nuklearmedizin und Interdisziplinäres Brustzentrum, \\ e Strahlentherapeutische Klinik und Interdisziplinäres Brustzentrum, \\ f Psychoonkologischer Dienst, \\ gTumorzentrum Berlin-Buch,

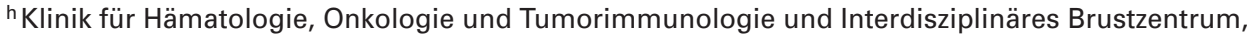 \\ i Medizinisches Controlling, HELIOS Klinikum Berlin-Buch, Germany
}

Das HELIOS Klinikum Berlin-Buch ist ein Krankenhaus der Maximalversorgung und Akademisches Lehrkrankenhaus der Charité Universitätsmedizin Berlin und liegt an der nordöstlichen Grenze der Hauptstadt. Die Frauenklinik Berlin-Buch hat eine langjährige Tradition in der Behandlung gynäkologischer Erkrankungen. Seit 2001 ist das Klinikum Berlin-Buch eine von jetzt 55 Kliniken im HELIOS-Konzern. Die Fachgruppe Gynäkologie und Geburtshilfe im HELIOS-Konzern hat unter der Leitung von Prof. Dr. M. Stark Initiativen ergriffen, um die Senologie bei HELIOS auf höchstem internationalen Stand zu etablieren. Seit Januar 2006 wird das Brustzentrum durch Herrn Prof. Dr. Michael Untch geleitet. Insgesamt werden in den HELIOS-Kliniken über 2000 Mammakarzinompatientinnen jährlich operiert. Die hohe Versorgungsqualität in der Behandlung dieser Patientinnen spielt bei Helios eine zentrale Rolle. Einige Kriterien dafür seien hier genannt:

- Steigerung der Anzahl brusterhaltender Operationen,

- Steigerung der Erkennung und Behandlung von Brustkrebs im Vorstadium (duktales Carcinoma in situ),

- primäre und sekundäre Brustrekonstruktion mittels Prothesen und Lappenplastiken,

- Etablierung einer Sprechstunde zur Beratung von Frauen aus Familien mit gehäuftem Auftreten von Mamma- und Ovarialkarzinom,

- Verbesserung der systemischen Therapie, insbesondere im Rahmen von kontrollierten nationalen und internationalen Studien,

- Einsatz neuer molekularbiologischer Erkenntnisse in der Diagnostik und Therapie des Mammakarzinoms,
- Verbesserung der Nachsorge, insbesondere Teilnahme an innovativen Studien in der Mammakarzinom-Nachsorge, die von Patientinnen zunehmend gefordert werden.

Etwa 2 Drittel der Patientinnen der Frauenklinik Berlin-Buch kommen aus der Bundeshauptstadt, das andere Drittel aus dem umgebenden Umland, insbesondere aus Brandenburg. An diesem Standort wurden schon sehr früh, bereits im Jahre 2000, Maßnahmen zur Verbesserung von Diagnostik und Therapie des Mammakarzinoms eingeführt.

Unter der Leitung von Frau PD Dr. Kettritz wurde eine Mammographiediagnostik-Einheit inklusive aller minimal-invasiven Biopsieverfahren etabliert. Die Sentinel-LymphknotenUntersuchung wurde eingeführt. Diese Methode wird in Kooperation mit der Nuklearmedizinischen Klinik unter Leitung von Prof. Dr. Dresel angeboten. In der Frauenklinik BerlinBuch werden seit 2001 Hospitationskurse für die SentinelLymphknoten-Untersuchung angeboten. Mittlerweile ist die Klinik unter den führenden in Deutschland, die die SentinelLymphknoten-Untersuchung vor neoadjuvanter Therapie durchführen (im Rahmen neoadjuvanter Studienkonzepte zusammen mit Prof. Dr. Kühn, Frauenklinik Esslingen und Dr. Bauerfeind, Universitätsfrauenklinik München-Großhadern). Das interdisziplinäre Brustzentrum im Klinikum Berlin-Buch wurde im August 2006 zertifiziert und im August 2007 durch die Deutsche Gesellschaft für Senologie, die Deutsche Krebsgesellschaft und den TÜV rezertifiziert. Seit Juli 2007 sind die Kliniken der Maximalversorgung in Berlin-Buch in einen Neubau umgezogen, für den der HELIOS-Konzern 240 Millionen Euro investiert hat. Es handelt sich um ein 1000-Betten-

\begin{tabular}{ll}
\hline KARGER & @ 2007 S. Karger GmbH, Freiburg \\
$\begin{array}{l}\text { Fax +49 76145207 14 } \\
\begin{array}{l}\text { E-mail Information@Karger.de } \\
\text { www.karger.com }\end{array}\end{array}$ & $\begin{array}{l}\text { Accessible online at: } \\
\text { www.karger.com/brc }\end{array}$
\end{tabular}


Haus mit modernster Ausstattung und angenehmer Atmosphäre für Patienten und Besucher. Zu der apparativen Ausstattung gehören zwei digitale Mammographie-Einheiten (Leitung Prof. Dr. J. Wildberger), eine PET-CT-Einheit in der Nuklearmedizinischen Klinik sowie eine neue Bestrahlungseinheit in der Klinik für Radiotherapie unter der Leitung des neu berufenen Chefarztes der Strahlentherapeutischen Klinik, Prof. Krempien. Jährlich werden 8000 Mammographien, 4000 Sonographien und 5000 MRTs in der diagnostischen Einheit durchgeführt. In der Pathologie (Leitung PD Dr. Th. Mairinger) werden 12000 Gewebeproben untersucht.

Die diagnostisch-radiologische Einheit ist eingebettet in das System der Screening-Mammographie in Berlin. Hierzu gibt es in der Bundeshauptstadt 4 Screening-Einheiten: Einheit 01, 13405 Berlin (zuständig für die Bezirke Spandau, Reinickendorf, Mitte), Einheit 02, 10437 Berlin (zuständig für die Bezirke Pankow, Lichtenberg, Marzahn-Hellersdorf), Einheit 03, 10967 Berlin (zuständig für die Bezirke Treptow-Köpenick, Neukölln, Friedrichshain-Kreuzberg) und Einheit 04, 12163 Berlin (zuständig für die Bezirke Termpelhof-Schöneberg, Steglitz-Zehlendorf, Charlottenburg-Wilmersdorf). Die Screening-Einheiten in der Bundeshauptstadt (Leitung Fr. Dr. L. Regitz-Jedermann) haben ihre Arbeit im Jahre 2006 bzw. 2007 aufgenommen und sind mittlerweile sehr gut von der Bevölkerung, den niedergelassenen Frauenärzten sowie den kooperierenden Brustzentren angenommen worden. Die Brustzentren beteiligen sich an den wöchentlichen Patientenbesprechungen in den Mammographie-Screening-Einheiten. Dabei werden die niedergelassenen Frauenärztinnen und Frauenärzte (Leiter des Berufsverbandes Dr. A. Scheffler) in das Screening-System, in die Therapieentscheidung und in die Nachbetreuung der Mammakarzinom-Patientinnen mit einbezogen.

In Berlin gibt es insgesamt 9 zertifizierte Brustzentren. Der Austausch findet im Rahmen von wissenschaftlichen Tagungen, Studienkooperationen und insbesondere auch DMP-Veranstaltungen und Patientinnen-Informationstagen statt. Die Kooperation, insbesondere mit der Universität Charité, wird im Rahmen von Studien und in einem Betreuungsprogramm zur Beratung von Frauen mit familiärem Mamma- und Ovarialkarzinom-Risiko durchgeführt. Diese Untersuchungen wurden über 6 Jahre von der Mildred-Scheel-Stiftung im Rahmen eines wissenschaftlichen Programmes unterstützt, mittlerweile sind die Leistungen dieser familiären Brust- und Eierstockkrebs-Sprechstunde in ein Versorgungsprogramm der Krankenkassen eingegangen. Die dabei gewonnenen Daten sollen dazu dienen, die Untersuchung und Betreuung von Frauen aus solchen Familien zu verbessern und Ratsuchende aus diesen Familien einer BRCA-Diagnostik zuzuführen, falls gewünscht.

Insgesamt werden im interdisziplinären Brustzentrum des Helios-Klinikums Berlin-Buch jährlich knapp 400 Eingriffe an der Brust, davon etwa 250 primäre Mammakarzinomeingriffe durchgeführt. Der Anteil an brusterhaltenden Therapien liegt beim pT1-Karzinom bei über $80 \%$ und bei pT2-Karzinomen bei über $60 \%$. Großer Wert wird auf die primäre systemische Therapie, insbesondere bei größeren Karzinomen und inflammatorischen Mammakarzinomen gesetzt. Dieses Vorgehen ist eingebettet in die nationale und internationale Studienlandschaft. Dabei hat sich unser Brustzentrum unter die Top 10 der rekrutierenden Kliniken bei neoadjuvanten und adjuvanten Studienkonzepten in Deutschland eingereiht (z. B. GeparQuattro-Protokoll in der Zusammenarbeit zwischen Arbeitsgemeinschaft Gynäkologische Onkologie und German Breast Group).

Es finden wöchentliche präoperative und postoperative interdisziplinäre Tumorboards statt. An diesen nehmen alle beteiligten Fachdisziplinen teil. Dabei wird sehr großer Wert darauf gelegt, dass Assistentinnen und Assistenten in der Weiterbildung die Fälle vorstellen und mit den anwesenden Fachärzten und Oberärzten diskutieren. Dies trägt ganz wesentlich zur Aus- und Weiterbildung bei. Die Kollegen der Poliklinik im HELIOS-Klinikum sind in die Therapie und Nachsorge von Brustkrebspatientinnen eng eingebunden und nehmen an den wöchentlichen Tumorboards teil. Einweisende Gynäkologinnen und Gynäkologen sind in den Tumorboards eingeladen, die Therapieempfehlungen mit den behandelnden Kollegen aus der Klinik aus ihrer Sicht zu besprechen. Die Therapieempfehlungen aus dem Tumorboard werden immer ausführlich und verständlich mit den Patientinnen besprochen. Das letzte Wort hat immer die betroffene Frau, und die Patientinnen haben selbstverständlich die Möglichkeit, die Meinung von weiteren Experten ihres Vertrauens einzuholen.

Die operative Therapie wird nach präoperativer Besprechung im Rahmen von Fallkonferenzen im zentralen OPTrakt des Neubaus durchgeführt. Die Einbettung in einen zentralen OP ermöglicht kürzeste Wege zwischen Station und OP-Saal sowie zwischen radiologischer Diagnostik, Nuklearmedizin, Pathologie und OP-Station. PatientinnenAnmeldung sowie Dokumentation erfolgt über ISH-med. Hierbei wird auch an Schnittstellen gearbeitet, um eine bessere Kommunikation mit dem Tumorzentrum Berlin-Buch (GTDS-System) zu ermöglichen. Über solche Schnittstellen können Auswertungen insbesondere zu den Schlüsselparametern der Nachsorge erfasst werden: intramammäre Rezidive, lokoregionale Rezidive, kontralaterale Mammakarzinomerkrankungen, Metastasierung, Tod. Dies sind auch die Schlüsselparameter, die in Zukunft die Güte eines Brustzentrums definieren werden.

Von den Sentinel-Lymphknoten wird in der Pathologie eine Schnellschnittuntersuchung durchgeführt. Die Versendung geschieht auf schnellstem Wege durch eine Rohrpostanlage, so dass die Schnellschnittzeiten deutlich verkürzt werden. Die vollständige Aufarbeitung der Sentinel-Lymphknoten erfolgt danach an Paraffinschnitten entsprechend den gängigen Bearbeitungsprotokollen. Die präoperative Markierung bei nicht palpablen Tumoren sowie bei Zustand nach neoadjuvanter Therapie inklusive Clipmarkierung ist Standard und wird in 
der radiologischen Diagnostik durchgeführt. Eine Präparateradiographie im Rahmen dieser Operationen ist obligat.

Einmalig in Deutschland, ist im interdisziplinären Brustzentrum in Berlin-Buch seit 2006 eine Brustkrankenschwester (breast nurse) als Vollzeitkraft ausschließlich für Brustkrebspatientinnen tätig. Als Bindeglied zwischen Patientin, Angehörigen, Ärzten und allen übrigen am Behandlungsprozess Beteiligten unterstützt und berät sie die Patientin und führt sie durch das Labyrinth von Diagnostik, Therapie und Nachsorge. Die Brustkrankenschwester sorgt für verständliche Informationen und koordiniert die Kommunikation zwischen der Patientin und dem Team des Interdisziplinären Brustzentrums. Als Bestandteil des Kernteams des interdisziplinären Brustzentrums nimmt sie regelmäßig an den Tumorkonferenzen teil.

Zur besseren Orientierung für die Patientinnen des interdisziplinären Brustzentrums wurde ein Patientenordner entworfen. Darin können alle Befunde zur Primärdiagnose, Operation, Systemtherapie, apparativen Untersuchungen und Nachsorgeuntersuchungen eingeheftet werden. Damit ist auch eine möglichst schnelle und offene Kommunikation zwischen niedergelassenen Ärzten und behandelnder Klinik gewährleistet.

Eine der Qualitätsoffensiven bei HELIOS zielt auf die Verkürzung der Zeiten in der Zustellung von Arztberichten an niedergelassene Ärzte, Verkürzung der Zeit bis zum Vorliegen des endgültigen histologischen Befundes der Operation und der axillären Lymphknoten inklusive der Östrogen- und Progesteronrezeptor- und HER2-neu-Diagnostik. Letztere soll im Rahmen eines Qualitätsprogramms mit den Pathologen primär mit der CISH-Diagnostik durchgeführt werden. Dies hilft zum einen, besser Patientinnen zu identifizieren, die tatsächlich von einer adjuvanten Herceptin-Therapie profitieren (und damit auch Kosten zu sparen) und vermeidet unnötige Therapien bei Patientinnen mit zweifelhaften Befunden. Die Mehrinvestition wird aus Hausmitteln getragen. Die Neuorientierung im Rahmen der Mammakarzinom-Nachsorge, die insbesondere auch von Patientinnen verlangt wird, wird bei HELIOS intensiv und konstruktiv diskutiert. Dabei werden insbesondere neue Studienkonzepte berücksichtigt.

Die systemische Therapie wird im Neubau interdisziplinär durchgeführt. Die Patientinnen werden in der Frauenklinik anhand der Vorschläge aus dem Tumorboard beraten. Die Therapiehoheit und Verantwortung bleibt in den Händen der Frauenärzte. Die Chemotherapie wird in einer interdisziplinä- ren Einheit in angenehmer, patientenfreundlicher Atmosphäre durchgeführt. Dies trägt wesentlich dazu bei, dass Kosten gespart werden, da nicht jede einzelne Abteilung ihre eigene Chemotherapie-Einheit hat. In unserem Brustzentrum führen wir zur Zeit folgende Studien zur neoadjuvanten und adjuvanten Therapie durch: GeparQuinto, ICE, GAIN, FACE, TEACH, ALLTO, Neo-ALLTO und CASA. Die Therapieentscheidung bei Patientinnen mit metastasierter Erkankung wird in unserem Brustzentrum ebenfalls interdisziplinär diskutiert. Dabei werden folgende Studien durchgeführt: Pelican, Athena, Alimta, Boehringer 1200.10 und LEAP.

Für unser Brustzentrum steht eine Psychoonkologin zur Verfügung, deren Leistungsspektrum von der Beratung und Information über supportive Einzelgespräche, Krisenintervention, tiefenpsychologisch fundierte Psychotherapie bis zu Angehörigengesprächen, Sterbebegleitung und Vermittlung von Selbsthilfegruppen und weiterführender Psychoonkologie reicht.

Für Studien und Dokumentation steht je eine Vollzeitkraft zur Verfügung. Eine intensive Zusammenarbeit besteht insbesondere mit den Studiengruppen Arbeitsgemeinschaft Gynäkologische Onkologie (AGO) und German Breast Group (GBG) sowie Westdeutsche Studiengruppe. Das Brustzentrum ist von Anfang an integriert in die Aktivitäten der Nordostdeutschen Gruppe für gynäkologische Onkologie (NOGGO), sowohl beim Mammakarzinom als auch beim Ovarial- und Zervixkarzinom.

Das Brustzentrum Berlin-Buch bietet für niedergelassene Ärztinnen und Ärzte sowie Kooperationspartner im DMPProgramm regelmäßige Fortbildungen an. Diese beziehen sich sowohl auf diagnostische Themen, wie Screening-Mammographie, minimal-invasive histologische Sicherung, als auch auf therapeutische Themen, wie operative Therapie, Sentinel-Lymphknoten-Untersuchung, neoadjuvante Therapie, neue endokrine und chemotherapeutische Behandlungskonzepte, familiäres Mamma- und Ovarialkarzinom, Nachsorge und psychoonkologische Betreuung. Regelmäßig, mindestens zweimal im Jahr, führt unser Brustzentrum Fortbildungsveranstaltungen speziell für Patientinnen durch. Die Themen werden hierbei so gewählt, dass Patientinnen und ihre Angehörigen die Sprache der Ärzte möglichst gut verstehen können.

Steigende Patientenzahlen belegen die Akzeptanz unseres Brustzentrums durch die Patientinnen und auch die zuweisenden Ärzte. 